Research Article

\title{
Application of New Sensor Technology in the Field of Education in the Era of Internet of Things
}

\author{
Haijun Chen $\mathbb{D}^{1},{ }^{1}$ Cong $M a \mathbb{D}^{2},{ }^{2}$ and Yiwei Wang $\mathbb{D}^{3}$ \\ ${ }^{1}$ School of Business, Wuzhou University, Wuzhou 543003, China \\ ${ }^{2}$ School of Economics and Management, Guangxi University of Science and Technology, Liuzhou 545006, China \\ ${ }^{3}$ School of Culture and Communication, Henan Finance University, Zhengzhou 450000, China
}

Correspondence should be addressed to Yiwei Wang; wangyiwei@hafu.edu.cn

Received 13 August 2021; Revised 18 September 2021; Accepted 7 October 2021; Published 26 October 2021

Academic Editor: Mu Zhou

Copyright (c) 2021 Haijun Chen et al. This is an open access article distributed under the Creative Commons Attribution License, which permits unrestricted use, distribution, and reproduction in any medium, provided the original work is properly cited.

In order to explore the intelligent education mode in the context of the Internet of Things, this paper combines the Internet of Things technology and sensor technology to improve the sensor technology and proposes a multisensor information fusion technology based on Kalman filtering. Moreover, this paper combines the wireless network technology to construct the system and structure, obtains the functional modules of the intelligent education system based on the Internet of Things technology and the new sensor technology, and analyzes the system realization process. In addition, this paper constructs a smart education system based on the Internet of Things and new sensors and designs experiments to verify the system. The research shows that the method proposed in this paper has good data transmission effect, can effectively improve the effect of intelligent education, and meet the actual needs of current education.

\section{Introduction}

After years of development, the Internet of Things is no longer a connection between things based on RFID technology, and its definition has been extended and changed very much. In general terms, the current Internet of Things refers to connecting all devices through Internet technology, allowing them to exchange data and information, and completing the intelligent management and control of devices [1]. More specifically, the Internet of Things acts as an information aggregation platform and provides a common language for the interaction of different devices and applications. The transmission of encrypted data is completed between the device and the Internet of Things platform. The Internet of Things integrates data from various devices and applications and realizes data interaction between applications through data analysis [2].

With the development and maturity of information technology and multimedia technology, classrooms have evolved from traditional multimedia classrooms to intelligent and intelligent classrooms [3]. It can be expected that with the maturity and popularization of the Internet of Things technology, mobile devices represented by smartphones, as well as terminal devices such as cameras and sensors, can extend and strengthen the various senses of classroom participants. At the same time, wearable devices can assist teachers and students in teaching activities to directly experience virtual reality/augmented reality (VR/AR) and assist classroom learning [4].

With the maturity and popularization of technologies such as artificial intelligence and deep learning, learning data will be analyzed using deep learning methods in the learning process to provide guidance or feedback for teaching behavior and provide analysis and prediction for teaching results. By analyzing the learning process data recorded by smart cameras, wearable devices, and various sensors, the smart classroom can automatically analyze the facial expressions and gestures of learning in real-time, so that teachers can get more teaching feedback information and improve teaching and learning. The purpose is to improve student learning efficiency.

As the most typical teaching environment application for the development of educational informatization, "smart 
classroom" has different understandings from different countries, experts, and scholars. The smart classroom is a new type of smart classroom that includes five dimensions: "presentation of teaching content," "classroom environment management," "teaching resource acquisition," "timely classroom interaction," and "environmental situational awareness." It is an intelligent learning environment. Concentrated embodiment, high-end multimedia and network classrooms and smart classrooms are able to satisfy administrators to intelligently control all the teaching equipment in the classroom, and at the same time, it can promote teaching activities between teachers and students through remote teaching and other methods. It is characterized by computer interaction, relying on smart space technology to improve and expand the functions that can be provided in the classroom; the smart classroom is an enhanced classroom based on electronic technology; the smart classroom can push different learning content to students according to the differences of different students and really do to personalized teaching. The entire classroom is people-oriented, and the effective interaction between people and smart devices promotes the achievement of learning goals; the function of the smart classroom is to use smart teaching equipment to collect and store the behavior data of students in teaching and to improve the teaching content through feedback information. Some smart devices that can quickly collect and find information can also be used to enhance the intelligence of the classroom. It can be found that the definition of the smart classroom is very broad, and the realization of different intelligent functions of the smart classroom depends on the corresponding different technical foundations.

This article combines the Internet of Things technology and new sensor technology to study the intelligence of the education field and combines the contemporary education needs to construct an intelligent education system to improve the effect of subsequent intelligent education.

\section{Related Work}

In the early stage of classroom construction, the data source of education and teaching was relatively single, the data structure was relatively single, and the main type is relational data. Moreover, the storage method is mainly based on relational databases, among which the more representative ones are MYSQL, Oracle, etc. With the rise of computers and the Internet and the development of the electronics industry, the application of computers in all walks of life, data sources have become widespread, and data types have become diverse. It is mainly divided into structured data and unstructured data. Databases used to store data are divided into two categories: relational databases and NoSQL (not only structured query language) databases. The application and development of these technologies have expanded the source of data. However, these data are stored independently in different systems. They are all data in a single field. There is no data circulation and data sharing, which has caused the phenomenon of data closure and formed a block of data [5]. Moreover, the source of the data is based on the transaction flow, so there is a lack of behavioral data, humanistic data, and social activity data associated with people. At the same time, due to technical limitations, these data have not been well utilized [6].

The high integration of sensors, collaborative sensing technology, mobile sensing technology [7], and other technologies are widely used in various fields to promote the development of sensing technology. Navigation is a typical application of location-based perception technology, which is mainly divided into two categories: car navigation and mobile phone navigation. In the early days, GPS- (Global Positioning System-) based location-aware technology achieved real-time recording and reporting of bus arrivals in modern urban traffic. Real-time information acquisition has improved travel convenience and made public travel more inclined to choose public transportation. In modern transportation systems, perception technology and intelligent technology are used to realize unmanned driving technology [8]. The development of mobile communication technology and wireless communication has played down the acquisition of geographic information. Even if the location of the other party is not known, instant communication can still be carried out. With the development of spatial information, it has emerged in various fields, especially situational awareness. In order to solve the problem that sensor devices in the Internet of Things need to manually set up the Internet, the context-aware dynamic discovery of things (CADDOT, Context-Aware Dynamic Discovery of Things) model [9], which can automatically identify, add and set sensor devices to expand perception range and perception data types. Pervasive perception is also a newly proposed concept. In intelligent sensing devices (mobile phones, tablets) with highly integrated sensors, the perception of smartphones has also become a research hotspot of data perception [10]. According to different perception objects and different application purposes, it can be roughly divided into five categories: physical environment perception, object information system identification perception, human body-related biological characteristics perception, spatial perception, and interactive information perception. The data included in physical environment perception is mainly structured data, and it is widely used in industry and agriculture. Object information system identification perception is widely used in logistics and other aspects, mainly for identification of objects. Spatial perception is the most widely used for location positioning. Interactive information perception has been widely used in applications such as personalized recommendation. Sensors with physiological related characteristics have also been widely used, and personal information data has been expanded to include personal information, personal behavior, personal physiological, and social activities and other related information [11].

In early research, foreign scholars believed that smart classrooms are classrooms enhanced by information technology [12]. At present, the concept of smart classroom has undergone significant changes. Foreign countries no longer define the concept of smart classroom from the aspect of classroom information technology, but from the learning environment [13]. The literature [14] pointed out that the classroom is a learning environment. Moreover, it is believed 
that the classroom not only includes all aspects of technology and resources but should also include various methods of education, and the classroom is a learning environment composed of teachers and learners. This literature incorporates the main body of teaching and learning into the study of the learning environment of the smart classroom. The literature [15] put forward the concept of an upside-down classroom. This teaching method gives students more freedom and further reflects the concept of education and teaching that students are the main body of learning.

\section{New Sensor Technology Based on the Internet of Things}

Sensor technology is one of the main contents of modern information technology. The sensor is a device that converts the amount of external environment information that can be felt into a required output signal through a set rule. It is generally composed of conversion components and sensitive components. The sensitive element refers to the part that can perceive external environmental information, and the conversion element refers to the part where the sensor transforms and outputs the external environmental information sensed by the sensitive element through certain rules.

The basic requirement for the static characteristics of the sensor is that when the input is 0 , the output is also 0 , or the output should maintain a certain corresponding relationship with the input. As shown in Figure 1, if the input of the sensor is $x$ and the output is $y$, then $y=f(x)$, and $x / y$ is preferably a constant proportional relationship [16].

Sensitivity and SN: sensitivity is the primary consideration when selecting a sensor. If the sensitivity required for measurement is not achieved, this sensor cannot be used. However, sensors with high sensitivity are susceptible to noise. In addition to environmental noise, there is also noise from the sensor itself [17].

Linear: there is a linear proportional relationship between input and output, which is called linear relationship. In fact, most sensors have a nonlinear relationship.

Hysteresis: when the input quantity increases to $X_{1}$, if the output quantity is $Y_{1}$, the input quantity continues to increase and then decreases to $X_{1}$, and the output quantity is $Y_{2}$ at this time. In fact, the output $Y_{1}$ and $Y_{2}$ are not equal, and there is a certain difference, which is a time lag. In this way, there is no one-to-one correspondence between input and output.

Environmental characteristics: the biggest influence on the sensor is the temperature in the surrounding environment. Moreover, many sensor materials choose semiconductors with high sensitivity and easy signal processing. However, semiconductors are sensitive to the environment, so special care must be taken when using them.

Stability: when sensors with ideal characteristics are added with the same input, the output is always the same. In actual use, the sensor changes with time, and the output may be different for the same input. During continuous work, temperature drift may also occur [18].

Accuracy degree: accuracy degree is used to evaluate the degree of excellence of the system. Accuracy is divided into

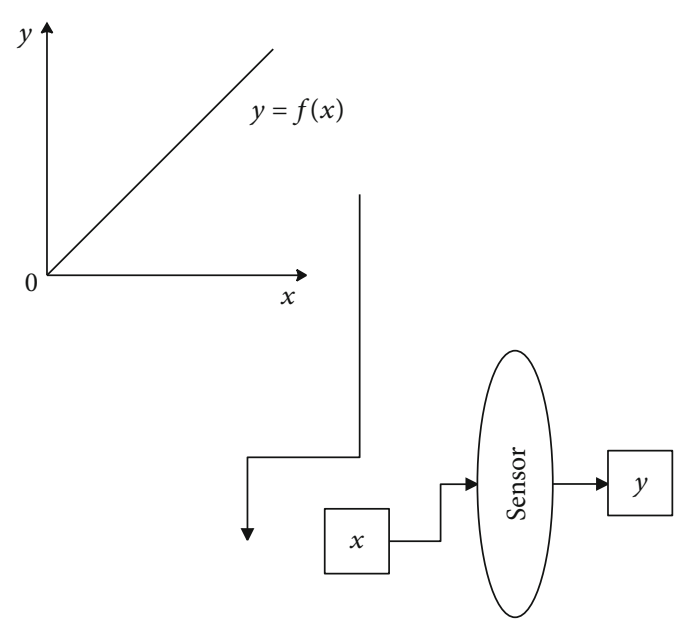

FIGURE 1: The relationship between input and output.

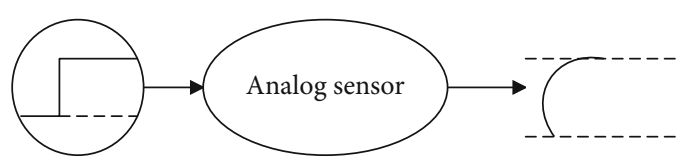

FIGURE 2: The relationship between input and response.

accuracy and precision. Accuracy refers to the deviation between the measured value and the true value, and correction is needed to correct this deviation. Precision refers to the same object, and each measurement will get a different measurement value, which is the discrete deviation.

The input signal to be detected by the sensor changes with time, and the characteristics of the sensor should be able to track the change of the input signal, so that an accurate input signal can be obtained. If the change is too fast, it may not be tracked. This is the response characteristic, that is, the dynamic characteristic. The dynamic characteristic is one of the important characteristics of the sensor. The relationship between sensor and response is shown in Figure 2.

Among the functional models of multisource information fusion systems, the JDL model and its evolutionary version occupy an important position, and it is currently a widely recognized model. The recommended information fusion model based on JDL is shown in Figure 3.

Level 0 signal/feature estimation: it estimates the state of the signal or feature. Signals and characteristics can be defined as data obtained from observations or measurements.

Level 1 entity estimation: it estimates the state or characteristics of the observed entity.

Level 2 situation estimation: it is an estimation of the actual observed environmental structure, that is, an estimation of the relationship between entities.

Level 3 impact estimation: it is an estimation of the availability and cost of signals, entities, and situational states.

Level 4 process estimation: the process of self-estimation of the system performance completed by comparing with the expected performance and efficiency.

As can be seen from the above figure, the recommended information model clearly divides the functions of each level 


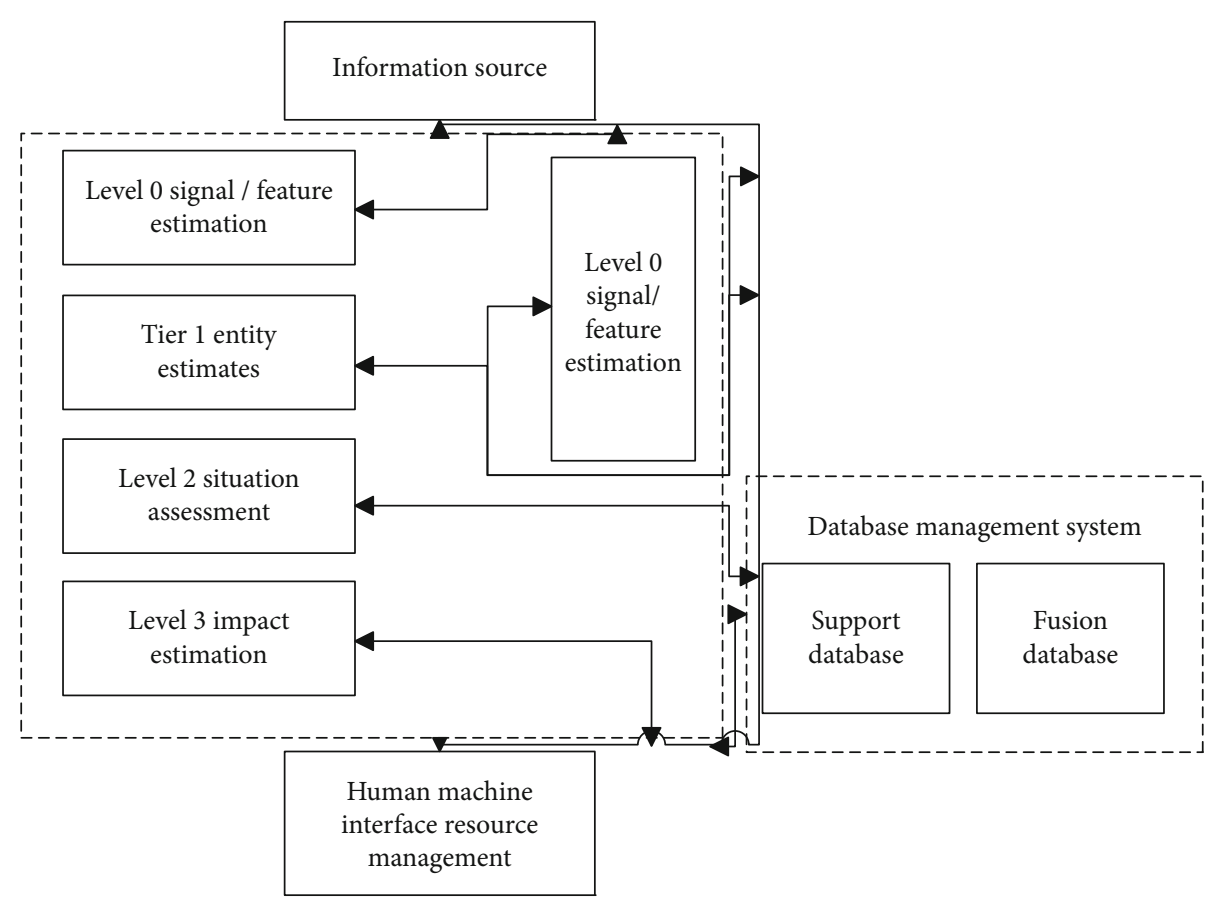

FIgURE 3: Recommended information fusion model.

of information fusion from the aspects of input data, model, output data, and reasoning type.

Multisensor information fusion is very different from previous single-sensor systems. The key is that the multisensor information of information fusion has a more complex form and can work at different information levels. There are many classification methods for data fusion. One is to classify the sensor data according to the degree of processing before it is sent to the data fusion processing center, which can be divided into sensor data fusion, central-level data fusion, and hybrid data fusion. The so-called hybrid data fusion means that it includes both sensor fusion and central-level fusion in its fusion process [19].

3.1. Sensor Data Fusion. Sensor data fusion is a distributed fusion structure. The basic principle is that different sensors collect the data of their respective observation targets and then input them to different local processors for local processing. After local processing, a local analysis result is formed. Finally, each local processor transmits different local estimates to the fusion center, and then the fusion center performs final processing on the data to form a global estimate.

It can be seen from the above that the sensor data fusion structure can perform simple partial processing on the data after the sensor collects the data, optimizes the data, and makes the system very simple, so it is widely used at present, and its structure is shown in Figure 4.

3.2. Central Fusion. Central fusion is also called centralized fusion, which means the fusion of original observation data. It transfers the data of each sensor to the data fusion center and performs data calibration, data association, track/pointtrack fusion, prediction, and tracking in the data fusion cen-

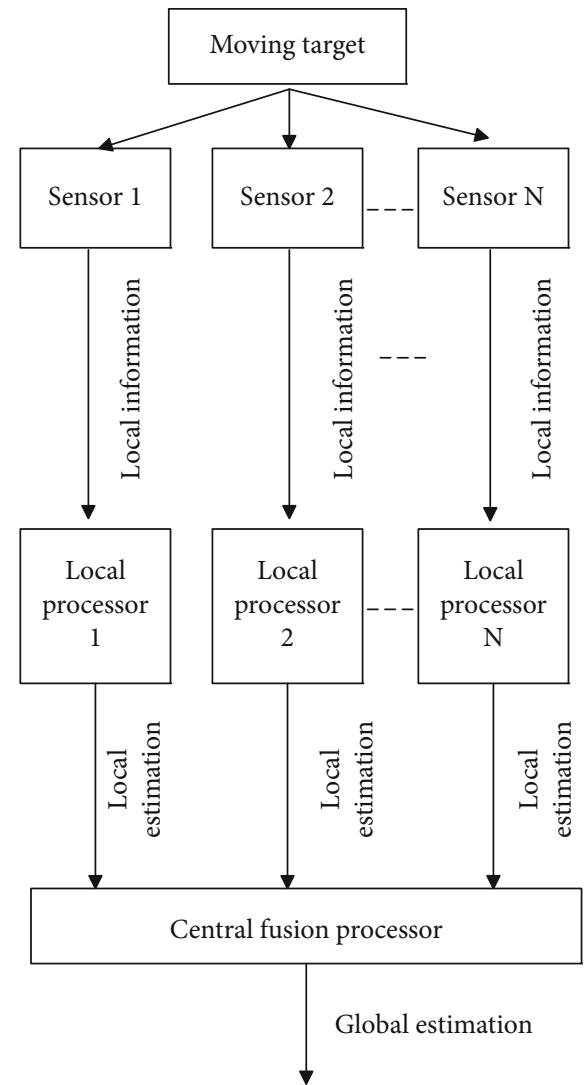

Figure 4: Sensor fusion structure model.

ter. Its structure is shown in Figure 5. If the data collected by each independent sensor can be accurately fused in the fusion center, the structure of the central fusion is the method that can obtain the most accurate observations. 


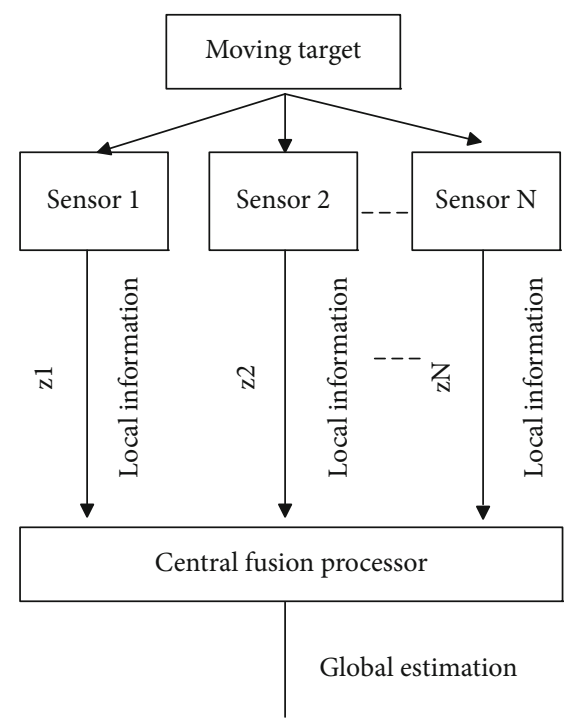

FIgURE 5: Central fusion structure model.

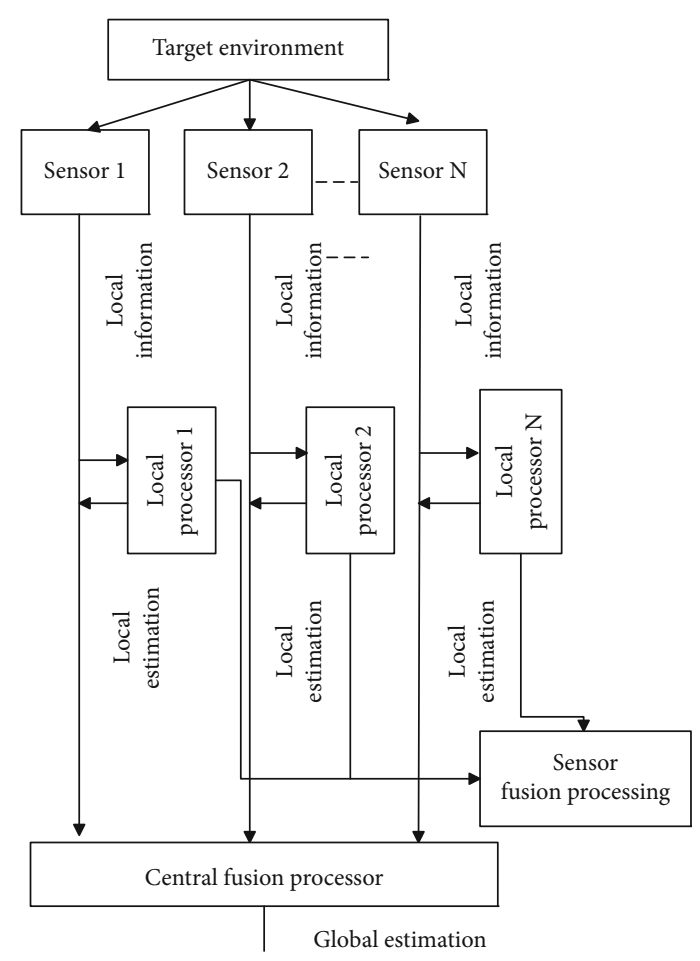

Figure 6: Hybrid fusion structure model.

However, under actual conditions, due to the large number of sensors, the data collected by some sensors may have serious errors that cannot be collected. Moreover, it is sometimes difficult to distinguish which sensors are collecting data from the same target. This makes the processing accuracy of the central-level data fusion structure higher, but the cost is also high and the stability is poor.

The hybrid fusion structure emphasizes the monitoring fusion process and effective scheduling data-level fusion, so it has strong adaptability, and takes into account the advantages of centralized fusion and distributed fusion structure, and has strong stability, as shown in Figure 6. However, this structure increases the complexity of data processing and needs to increase the data transmission rate, so it is timeconsuming in communication and calculations [20].

As an intelligent education system, angle sensors can be installed in the classroom to detect the rotation joints of the camera device, so that the angle between the joints can be accurately obtained. After the angle is obtained, the calculation can be performed according to Equation (1) to obtain the pose equation of the wrist joint [21].

$$
\begin{gathered}
{ }^{0} T_{3}={ }^{0} T_{1}{ }^{1} T_{2}{ }^{2} T_{3}, \\
{ }^{0} T_{1}=\left[\begin{array}{cccc}
c \theta_{1} & 0 & -s \theta_{1} & 0 \\
s \theta_{1} & 0 & c \theta_{1} & 0 \\
0 & -1 & 0 & 0 \\
0 & 0 & 0 & 1
\end{array}\right] T_{2}=\left[\begin{array}{cccc}
c \theta_{2} & -s \theta_{2} & 0 & a_{2} c \theta_{2} \\
s \theta_{2} & c \theta_{2} & 0 & a_{2} c \theta_{2} \\
0 & 0 & 1 & 0 \\
0 & 0 & 0 & 1
\end{array}\right],
\end{gathered}
$$

$$
{ }^{2} T_{3}=\left[\begin{array}{cccc}
c \theta_{3} & -s \theta_{3} & 0 & a_{3} c \theta_{3} \\
s \theta_{3} & c \theta_{3} & 0 & a_{3} c \theta_{3} \\
0 & 0 & 1 & 0 \\
0 & 0 & 0 & 1
\end{array}\right] .
$$

$c \theta_{1}=\cos \theta_{1}, s \theta_{1}=\sin \theta_{1}$, and $a_{2}$ are the length of the big arm, and $a_{3}$ is the length of the forearm.

That is, as long as the value of $\theta_{1}, \theta_{2}, \theta_{3}$ is known, the pose of the wrist relative to the origin of the shoulder joint can be calculated according to Formula (1).

In actual working conditions, the data collected by the sensor may contain errors due to environmental interference, and the data collected by a traditional single sensor loses the characteristics after the information combination.

Kalman filtering has a good filtering effect in the field of linear filtering, especially the optimal state estimation can be obtained in the filtering process under the assumption of linear Gaussian white noise. Because the Kalman filtering method is simple and the linear filtering effect is good, it has been widely used in various fields, especially in the application of target tracking.

A discrete-time system described by the vector difference equation is considered. Due to the influence of Gaussian noise in the system, the state equation of the system is

$$
X(k+1)=F(k) X(k)+G(k) u(k)+v(k), k=0,1,2, \cdots .
$$

In the formula, $X(k) \in R$ is the state vector and $u(k) \in R$ is the known input vector. $v(k) \in R$ is the white noise sequence of the mean Gaussian process, and its covariance is

$$
E\left[\left(v(k) v(k)^{T}\right)\right]=Q(k) .
$$

The measurement equation of the system is

$$
z(k)=H(k) X(k)+w(k) .
$$




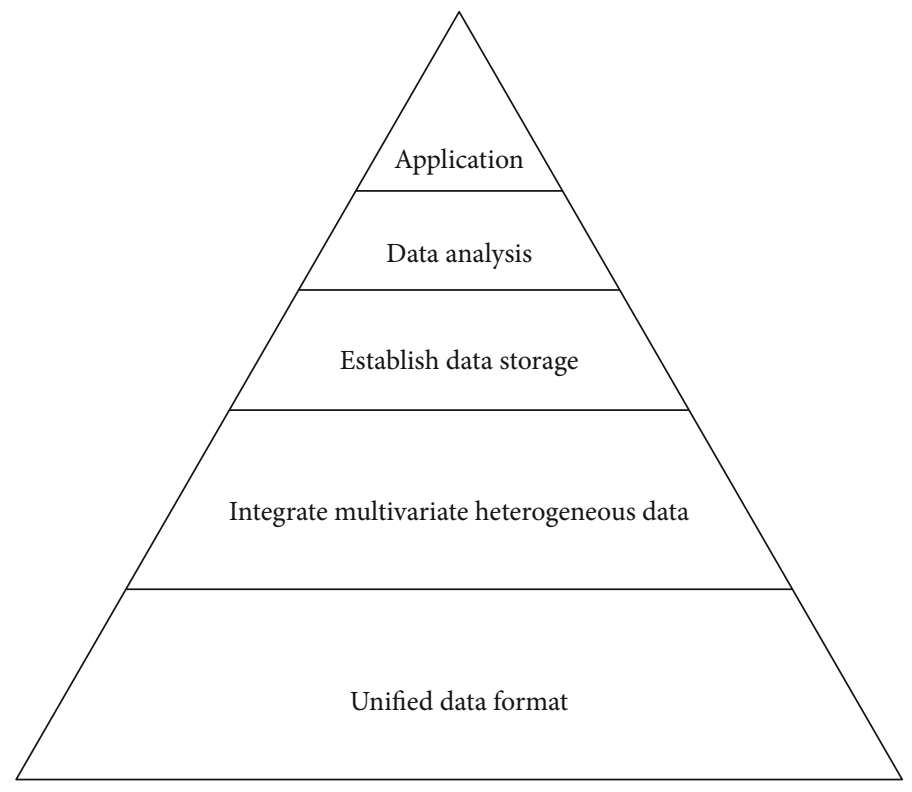

Figure 7: Multisource heterogeneous data processing model.

In the formula, $z(k) \in R$ is the observation vector, $w(k)$ $\in R$ is the zero Gaussian noise sequence with mean value, and its covariance is

$$
E\left[\left(w(k) w(k)^{T}\right)\right]=R(k) .
$$

The matrices $F(k), G(k), H(k), Q(k)$, and $R(k)$ are assumed to be known and may change over time. That is, the system may change with time, and the noise may also be non-stationary random noise.

Kalman filtering has two types of algorithms: continuous and discrete. Among them, the discrete algorithm is currently widely used in the state estimation of linear systems.

The algorithm flow of Kalman filter is as follows: filter estimation [22]:

$$
\widehat{X}(k+1 \mid k+1)=F \widehat{X}(k \mid k)+K(k+1)[Z(k)-H F \widehat{X}(k \mid k)] .
$$

Filter gain:

$$
X(k+1)=P(k+1 \mid k) H^{T}\left[H P(k+1 \mid k) H^{T}+R(k)\right]^{-1} .
$$

Single-step prediction error covariance matrix:

$$
P(k+1 \mid k)=F P(k \mid k) F^{T}+\Gamma Q(k) \Gamma^{T} .
$$

Filtering error covariance matrix:

$$
P(k+1 \mid k+1)=[I-K(k+1) H] P(k+1 \mid k) .
$$

It should be pointed out that $P(k+1 \mid k+1)$ can be regarded as the performance evaluation of Kalman filtering, because:

$$
\begin{aligned}
P(k+1 \mid k+1)= & E[(X(k+1)-\widehat{X}(k+1 \mid k+1))(X(k+1) \\
& \left.-X \wedge(k+1 \mid k+1))^{T}\right] .
\end{aligned}
$$

It can be seen from the Kalman filter process that the algorithm process of Kalman filter is actually a set of recursive formulas. The calculation process is a process of continuous prediction and correction according to the set initial value, state equation, and prediction equation. First, according to the state estimation at the previous moment, the next prediction value at the observation moment is obtained from the state equation; then, the correction value of the prediction value is calculated by combining the current real-time observation value and information, so as to obtain the optimal estimated value and complete the prediction.

In the design process of the Kalman filter, an important step is to determine the state vector of the filter, because the state vector often has an important influence on the entire algorithm. In the algorithm design process, considering the derivative relationship between speed and acceleration, speed can be regarded as a state vector. For the selection of another state vector, since once the deviation value of the acceleration is determined, the acceleration can be accurately estimated, so this deviation value can be selected as another state vector instead of directly estimating the acceleration.

We set

$$
\begin{aligned}
& v=f(x)+w_{v}, \\
& a=a(x)-\rho+w_{a} .
\end{aligned}
$$

In formulas (13) and formula (14), $v$ is the measured value of the speed sensor, $a$ is the measured value of the acceleration sensor, $w_{v}$ and $w_{a}$ are the noise interference of the speed sensor and acceleration sensor, respectively, and 


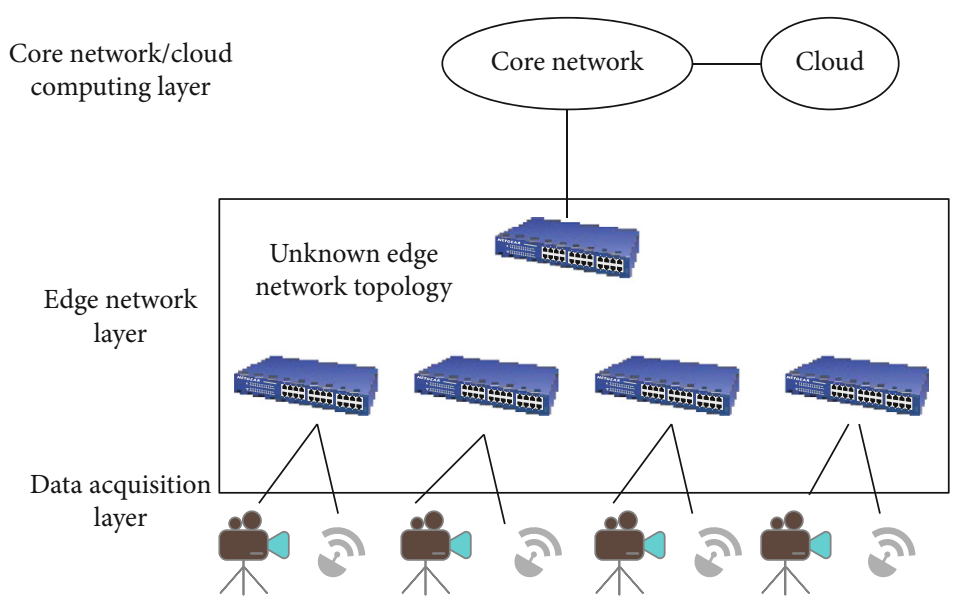

Figure 8: Edge big data processing model based on IoT cloud computing.

$\rho$ is the measured deviation value of the assumed acceleration sensor.

The matrix equation is established as follows:

$$
\begin{aligned}
{\left[\begin{array}{l}
v \\
\rho
\end{array}\right] } & =\left[\begin{array}{cc}
0 & -1 \\
0 & 0
\end{array}\right]\left[\begin{array}{l}
v \\
\rho
\end{array}\right]+\left[\begin{array}{l}
1 \\
0
\end{array}\right] a+\left[\begin{array}{c}
w_{a} \\
0
\end{array}\right], \\
v & =[10]\left[\begin{array}{l}
v \\
\rho
\end{array}\right]+w_{v} .
\end{aligned}
$$

Since the state vector selects the speed value and the deviation value from the acceleration, respectively, $x=$ $\left[\begin{array}{ll}v & \rho\end{array}\right]^{T}$, and the sampling time interval of the system is $\Delta t$, and

$$
x(k+1)=x(k)+\Delta t \cdot a .
$$

By combining formula (15) with formula (13), after sorting, we get

$$
x(k+1)=\left[\begin{array}{cc}
1 & -\Delta t \\
0 & 1
\end{array}\right] x(k)+\left[\begin{array}{c}
\Delta t \\
0
\end{array}\right] a(k)+\left[\begin{array}{c}
w_{a}(k) \cdot \Delta t \\
0
\end{array}\right] .
$$

After completing the design of the Kalman filter, the simulation environment is designed according to the known algorithm flow.

3.3. Selection of Simulation Tools. The selection of simulation tools should take into account the simplicity of algorithm writing and the ability to intuitively generate simulation results. Since the Kalman filter algorithm involves matrix operations and recursive operations, the MATLAB tool, which has powerful numerical analysis and matrix operations, and can intuitively generate graphs, is selected for simulation in this design.

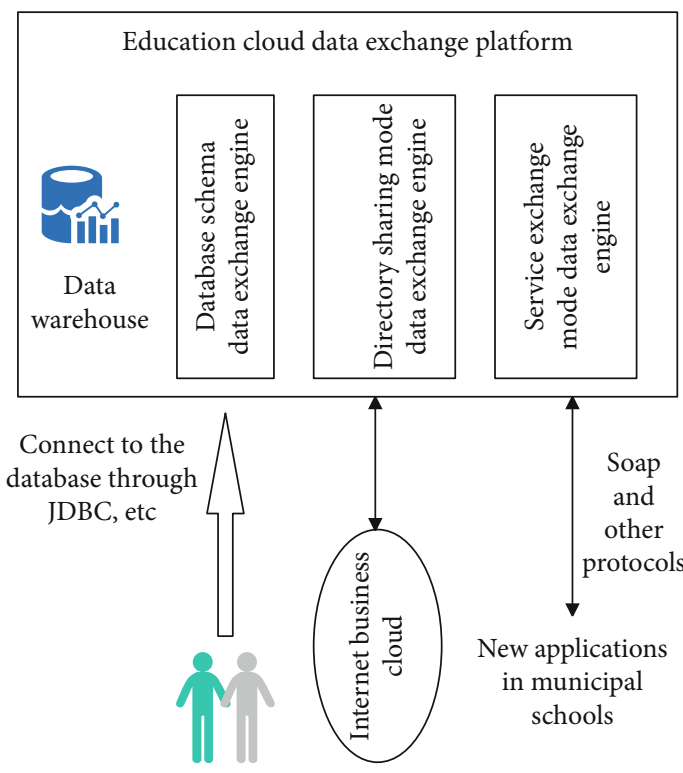

The school is using third-party applications such as library, all-in-one card and other systems

Figure 9: Data exchange function of education platform based on wireless network.

3.4. Determination of the Initial Value of the Filter. After determining the simulation tool to be used, it is necessary to program and simulate the filter. In order to find the derivative more convenient, and to control the upper and lower boundaries of the function, we can choose the sine function and cosine function for simulation, as follows:

$$
\begin{aligned}
& v=\sin (4 t)+w_{v}, \\
& a=4 \cos (4 t)-5+w_{a} .
\end{aligned}
$$

Formula (18) is derived from formula (17) and obtained by setting the deviation value. $w_{v}$ and $w_{a}$ represent the variance values of the velocity function and acceleration function noise, respectively, and the values of $w_{v}$ and $w_{a}$ can be 


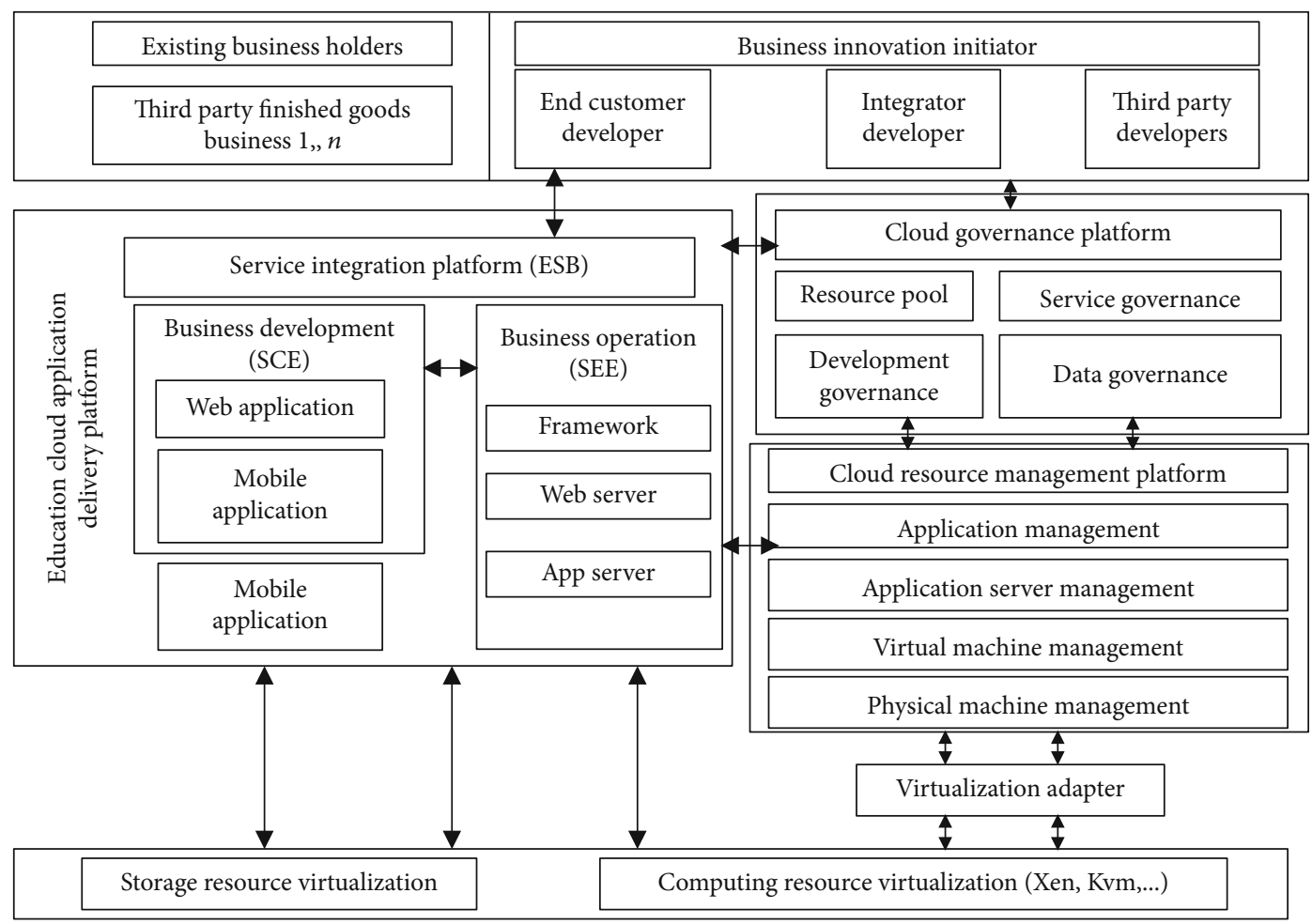

FIGURE 10: The framework of an intelligent education system based on the Internet of Things and new sensors.

set to 0.04 and 1 , respectively. When the time interval of the system is set to $\Delta t=0.01 \mathrm{~s}$, the matrix function is as follows:

$$
\begin{gathered}
f=\left[\begin{array}{cc}
1 & -\Delta t \\
0 & 1
\end{array}\right], F=\left[\begin{array}{ll}
\Delta t & 0
\end{array}\right]^{T}, p=\left[\begin{array}{ll}
0 & 1 \\
1 & 0
\end{array}\right], H=\left[\begin{array}{ll}
1 & 0
\end{array}\right], Q=\left[\begin{array}{rr}
\Delta t^{2} & 0 \\
0 & 0
\end{array}\right], \\
\operatorname{sgm} \Gamma=0.2, x=\left[\begin{array}{ll}
0 & 0
\end{array}\right]^{T} .
\end{gathered}
$$

Multisource heterogeneous data has the characteristics of large quantity, many data types, low value density, and authenticity. In order to realize the full application of multisource heterogeneous data, effectively carry out systematic analysis and data mining of multisource heterogeneous data, and obtain higher use value, this paper summarizes the multisource heterogeneous data processing requirements into a five-layer model, as shown in Figure 7.

\section{Smart Education System Based on Internet of Things and New Sensor Technology}

Figure 8 shows the processing model of big data at the network edge based on cloud computing. The model is divided into data collection layer, edge network layer, core network, and cloud computing layer. The data collection layer is responsible for collecting data, which is transmitted via the edge network and the core backbone network to the cloud computing center for processing. Among them, the edge network will be quite different according to different application scenarios. In this paper, it is assumed that the topology of the edge network is unknown.
The data exchange function of the education cloud platform can solve the problem of interconnection of business data generated within the smart class card system and external data such as education cloud, Internet commercial cloud, and school in-use information system. The data source of data exchange includes not only the business data generated by the smart class card system but also a large amount of event data using the smart class card system. Based on these two types of data sources, it satisfies the data query and retrieval, data mining, statistical analysis, and in-depth analysis. Figure 9 shows an example of the data exchange function of the education cloud platform.

As shown in Figure 10 is the overall architecture of the smart cloud platform, then all the subsystems, such as the teaching resource sharing subsystem, the educational administration system, and the all-in-one card system, will run on the service integration platform. Each application developer conducts unified planning to share and manage school, student personal information, and other data.

After constructing an intelligent education system based on the Internet of Things and new sensors, the performance of the system is verified. This article carries on simulation experiment through this Matlab, evaluates the performance of the intelligent education system through many groups of experiments, and counts the data transmission speed and teaching effect; the statistical result is shown in Table 1.

It can be seen from the above research that the method proposed in this paper has a good data transmission effect, can effectively improve the effect of intelligent education, and meet the actual needs of current education. 
TABLE 1: Performance evaluation of intelligent education system based on the Internet of Things and new sensors.

\begin{tabular}{|c|c|c|c|c|c|}
\hline Number & Transmission speed (ms) & System performance & Number & Transmission speed (ms) & System performance \\
\hline 1 & 143.45 & 76.51 & 26 & 134.23 & 75.39 \\
\hline 2 & 113.42 & 88.45 & 27 & 119.01 & 77.73 \\
\hline 3 & 100.33 & 74.88 & 28 & 143.80 & 80.42 \\
\hline 4 & 106.09 & 77.87 & 29 & 142.81 & 79.10 \\
\hline 5 & 113.67 & 77.38 & 30 & 121.02 & 75.02 \\
\hline 6 & 146.11 & 76.42 & 31 & 151.60 & 76.14 \\
\hline 7 & 148.37 & 86.08 & 32 & 122.74 & 83.42 \\
\hline 8 & 130.98 & 86.80 & 33 & 101.36 & 88.04 \\
\hline 9 & 141.18 & 95.17 & 34 & 107.95 & 96.79 \\
\hline 10 & 106.53 & 91.82 & 35 & 134.78 & 88.74 \\
\hline 11 & 144.50 & 92.93 & 36 & 138.67 & 92.91 \\
\hline 12 & 112.72 & 77.97 & 37 & 131.45 & 90.50 \\
\hline 13 & 99.11 & 77.21 & 38 & 106.24 & 85.88 \\
\hline 14 & 143.73 & 90.97 & 39 & 149.93 & 91.22 \\
\hline 15 & 122.87 & 79.51 & 40 & 106.84 & 79.17 \\
\hline 16 & 147.93 & 88.36 & 41 & 112.80 & 75.14 \\
\hline 17 & 143.05 & 95.58 & 42 & 107.42 & 87.91 \\
\hline 18 & 105.97 & 85.13 & 43 & 120.06 & 75.27 \\
\hline 19 & 112.06 & 85.46 & 44 & 122.07 & 88.08 \\
\hline 20 & 140.26 & 77.05 & 45 & 114.44 & 74.16 \\
\hline 21 & 98.70 & 78.29 & 46 & 148.19 & 78.95 \\
\hline 22 & 115.72 & 88.06 & 47 & 102.28 & 96.76 \\
\hline 23 & 131.77 & 78.57 & 48 & 126.43 & 83.35 \\
\hline 24 & 133.41 & 77.19 & 49 & 139.90 & 81.90 \\
\hline 25 & 110.05 & 74.91 & 50 & 113.15 & 91.13 \\
\hline
\end{tabular}

\section{Conclusion}

With the further in-depth integration of computer technology, information technology, Internet technology, artificial intelligence, and other technologies and education, the appearance of smart education will undergo earth-shaking changes. This article discusses the basic principles and related knowledge of sensor technology and multisensor information fusion theory, as well as the application of Kalman filter in sensor data fusion, including the definition, classification, characteristics, and selection of sensors.

This article focuses on the analysis of the business needs of the cloud application platform. The cloud application platform of the smart campus requires basic platform construction, smart portal construction, smart teaching construction, and smart management construction. At the same time, this paper mainly elaborates the construction plan of the smart campus cloud service network platform and implements it. In addition, this paper constructs a smart education system based on the Internet of Things and new sensors and designs experiments to verify the system. Through the research results, it can be seen that the method proposed in this paper has a good data transmission effect, can effectively improve the effect of intelligent education, and meet the actual needs of current education.

\section{Data Availability}

The labeled dataset used to support the findings of this study are available from the corresponding author upon request.

\section{Conflicts of Interest}

The authors declare no competing interests.

\section{Acknowledgments}

This study is sponsored by Henan Finance University.

\section{References}

[1] S. M. Chaware, S. Dighe, A. Joshi, N. Bajare, and R. Korke, "Smart garbage monitoring system using internet of things (IoT)," Ijireeice, vol. 5, no. 1, pp. 74-77, 2017.

[2] W. Tushar, N. Wijerathne, W. T. Li et al., "Internet of things for green building management: disruptive innovations through low-cost sensor technology and artificial intelligence," IEEE Signal Processing Magazine, vol. 35, no. 5, pp. 100-110, 2018.

[3] J. H. Abawajy and M. M. Hassan, "Federated internet of things and cloud computing pervasive patient health monitoring system," IEEE Communications Magazine, vol. 55, no. 1, pp. 4853, 2017. 
[4] D. He, R. Ye, S. Chan, M. Guizani, and Y. Xu, "Privacy in the internet of things for smart healthcare," IEEE Communications Magazine, vol. 56, no. 4, pp. 38-44, 2018.

[5] P. P. Ray, "Internet of things for smart agriculture: technologies, practices and future direction," Journal of Ambient Intelligence and Smart Environments, vol. 9, no. 4, pp. 395-420, 2017.

[6] Y. A. Qadri, A. Nauman, Y. B. Zikria, A. V. Vasilakos, and S. W. Kim, "The future of healthcare internet of things: a survey of emerging technologies," IEEE Communications Surveys \& Tutorials, vol. 22, no. 2, pp. 1121-1167, 2020.

[7] B. H. Dobkin, "A rehabilitation-internet-of-things in the home to augment motor skills and exercise training," Neurorehabilitation and Neural Repair, vol. 31, no. 3, pp. 217-227, 2017.

[8] J. Yao and N. Ansari, "Caching in energy harvesting aided internet of things: a game-theoretic approach," IEEE Internet of Things Journal, vol. 6, no. 2, pp. 3194-3201, 2019.

[9] J. E. Siegel, S. Kumar, and S. E. Sarma, "The future internet of things: secure, efficient, and model-based," IEEE Internet of Things Journal, vol. 5, no. 4, pp. 2386-2398, 2018.

[10] M. A. Abd-Elmagid, N. Pappas, and H. S. Dhillon, "On the role of age of information in the internet of things," IEEE Communications Magazine, vol. 57, no. 12, pp. 72-77, 2019.

[11] A. Sheth, U. Jaimini, and H. Y. Yip, "How will the internet of things enable augmented personalized health?," IEEE Intelligent Systems, vol. 33, no. 1, pp. 89-97, 2018.

[12] G. J. Joyia, R. M. Liaqat, A. Farooq, and S. Rehman, "Internet of medical things (IOMT): applications, benefits and future challenges in healthcare domain," The Journal of Communication, vol. 12, no. 4, pp. 240-247, 2017.

[13] N. Kshetri, "The evolution of the internet of things industry and market in China: an interplay of institutions, demands and supply," Telecommunications Policy, vol. 41, no. 1, pp. 49-67, 2017.

[14] S. Siboni, V. Sachidananda, Y. Meidan et al., "Security testbed for internet-of-things devices," IEEE Transactions on Reliability, vol. 68, no. 1, pp. 23-44, 2019.

[15] Y. Yang, M. Zhong, H. Yao, F. Yu, X. Fu, and O. Postolache, "Internet of things for smart ports: technologies and challenges," IEEE Instrumentation \& Measurement Magazine, vol. 21, no. 1, pp. 34-43, 2018.

[16] Z. Li, Y. Liu, A. Liu, S. Wang, and H. Liu, "Minimizing convergecast time and energy consumption in green internet of things," IEEE Transactions on Emerging Topics in Computing, vol. 8, no. 3, pp. 797-813, 2020.

[17] P. P. Ray, "A survey on internet of things architectures," Journal of King Saud University-Computer and Information Sciences, vol. 30, no. 3, pp. 291-319, 2018.

[18] M. Mayer and A. J. Baeumner, "A megatrend challenging analytical chemistry: biosensor and chemosensor concepts ready for the internet of things," Chemical Reviews, vol. 119, no. 13, pp. 7996-8027, 2019.

[19] M. Saez, F. P. Maturana, K. Barton, and D. M. Tilbury, "Realtime manufacturing machine and system performance monitoring using internet of things," IEEE Transactions on Automation Science and Engineering, vol. 15, no. 4, pp. 17351748, 2018.

[20] V. Jagadeeswari, V. Subramaniyaswamy, R. Logesh, and V. Vijayakumar, "A study on medical internet of things and big data in personalized healthcare system," Health information science and systems, vol. 6, no. 1, pp. 1-20, 2018.
[21] S. Smys, A. Basar, and H. Wang, "Hybrid intrusion detection system for internet of things (IoT)," Journal of ISMAC, vol. 2, no. 4, pp. 190-199, 2020.

[22] I. Butun, P. Österberg, and H. Song, "Security of the internet of things: vulnerabilities, attacks, and countermeasures," IEEE Communications Surveys \& Tutorials, vol. 22, no. 1, pp. 616644, 2020. 